\title{
Sensitive and Reproducible Photocatalytic Activity Evaluation Instrument for Transparent Coatings
}

\author{
Masayuki Kamei \\ National Institute for Materials Science, Tsukuba, Japan. \\ Email: kamei.masayuki@nims.go.jp \\ Received September $28^{\text {th }}, 2011$; revised December $2^{\text {nd }}, 2011$; accepted December $10^{\text {th }}, 2011$
}

\begin{abstract}
A new photocatalytic activity (PC) measurement instrument based on the measurement of the photo-induced reduction of Ag ions was proposed. The feature of this system is to perform "ultraviolet irradiation for PC activation" and "Ag film thickness determination for PC evaluation” simultaneously and automatically. Realizing a PC measurement system with high sensitivity, wide dynamic range, easy operation and reproducibility, which is especially suited for the PC measurement of the coatings on transparent substrates.
\end{abstract}

Keywords: $\mathrm{TiO}_{2}$; Coatings; Photo-Catalysis

\section{Introduction}

Since the discovery of the photosensitizing effect of $\mathrm{TiO}_{2}$ electrode on the electrochemical decomposition of water [1], $\mathrm{TiO}_{2}$ has attracted significant attention, and the photocatalytic properties of $\mathrm{TiO}_{2}$ has become a major area of intensive research. $\mathrm{TiO}_{2}$ has shown interesting phenomena, such as photo-induced oxidation and reduction, and photo-induced hydrophilic/ hydrophobic switching [2-5] and has been widely considered as the most promising photocatalytic material. For the practical use of this photocatalyst, coating technology of $\mathrm{TiO}_{2}$ is the key technology and has intensively been studied for achieving higher photocatalytic activity [6-8]. For this purpose, reproducible and high-throughput photocatalytic activity evaluation for coatings is necessary and several methods have been proposed. The effective surface area of the photocatalytic coatings is very small compared with that of the powders and hence the photocatalytic activity measurement for coatings should be not only reproducible but also highly sensitive. Photocatalytic reduction of Ag ions resulted in Ag film formation on the surface of the photo catalyst [9] and this phenomenon was used for the photocatalytic activity evaluation $[8,9]$. This method is very sensitive and applicable for the evaluation of the photocatalytic activity of the coatings. However, a well-trained operator is necessary for the reproducible measurement at high throughput since this method requires "ultraviolet irradiation" and "Ag film thickness determination" to be alternated [8]. In this study, we propose a modified Ag photoreduction method, which is capable of performing "ultraviolet irradiation" and "Ag film thickness determination" simultaneously and automatically. Accordingly, sensitive, reproducible and high-throughput photocatalytic activity evaluation method especially for transparent coatings was realized.

\section{Experimental}

Three kinds of $\mathrm{TiO}_{2}$ films coated on quartz substrates were used to demonstrate the potential of the new photocatalytic activity measurement method. A pulse-powered sputtering apparatus, which is described elsewhere in detail [7], was applied for coating the $\mathrm{TiO}_{2}$ films. The deposition conditions for the three kinds of films are listed in Table 1. The crystalline structures of the films were evaluated with an x-ray diffractometer (RIGAKU RINT).

Table 1. Sputter deposition conditions for the three kinds of $\mathrm{TiO}_{2}$ coatings.

\begin{tabular}{|c|c|c|c|c|c|c|c|}
\hline & substrate & temperature & power & $\mathrm{O}_{2}$ & pressure & thickness & time \\
\hline A & quartz & not heated 300 - $360 \mathrm{~K}$ & $5000 \mathrm{~W}$ & $12 \mathrm{sccm}$ & $0.5 \mathrm{~Pa}$ & $410 \mathrm{~nm}$ & $20 \mathrm{~min}$. \\
\hline $\mathrm{C}$ & quartz & heated $573 \mathrm{~K}$ & $5000 \mathrm{~W}$ & $12 \mathrm{sccm}$ & $0.5 \mathrm{~Pa}$ & $400 \mathrm{~nm}$ & $20 \mathrm{~min}$. \\
\hline
\end{tabular}


The schematic drawing of the photocatalytic activity measurement apparatus is shown in Figure 1. The $\mathrm{TiO}_{2}$ film coated quarts glass is placed in a quartz cell and the cell was filled with aqueous $\mathrm{AgNO}_{3}$ solution of $0.1 \mathrm{~mol} / \mathrm{l}$. The lights from the D2 lamp and Xe lamp was mixed by a half mirror and irradiated on the surface of the $\mathrm{TiO}_{2}$ coating. The ultraviolet light intensity measured by an ultraviolet photometer (MINOLTA UM-10 + UM-360) at the sample was $0.03 \mathrm{~mW} / \mathrm{cm}^{2}$. Note that the irradiation should be done from the coated side of the quartz glass. The Ultraviolet lights with the wavelength smaller than $380 \mathrm{~nm}$ act as excitation light for the photocatalytic reaction and adsorbed with traveling through the $\mathrm{TiO}_{2}$ coating. The lights with the wavelength larger than $380 \mathrm{~nm}$ travel through the $\mathrm{TiO}_{2}$ coating, the substrate, the cell and finally reaching the spectrometer, which is capable of measuring the optical transmittance of the sample.

On the surface of the $\mathrm{TiO}_{2}$ coating, the ultraviolet irradiation induced photocatalytic reduction of the Ag ions occurs and resulting in the Ag film formation on the surface of the $\mathrm{TiO}_{2}$ coating. The optical transmittance of the sample gradually decreases with the growth of the Ag film on the surface. The higher the photocatalytic activity of the coating is, the quicker the decrease of the optical transmittance becomes. Hence by recording the transition curve of optical transmittance of the sample (transmittance vs time), the photocatalytic activity of the coatings can be evaluated. The optical transmittance was measured every 12 seconds and recorded automatically by the optical spectrometer (Shimadzu Multispec 1500). The measurement time was set to 120 minutes for one sample, resulting in 600 points for one transition curve. All the operator has to do for measuring one sample is to put the sample in the quartz cell filled with aqueous $\mathrm{AgNO}_{3}$ and set it on the photometer and start the measurement software. Hence this method is reproducible (not dependent on the skill of the operator) as well as easy operation.

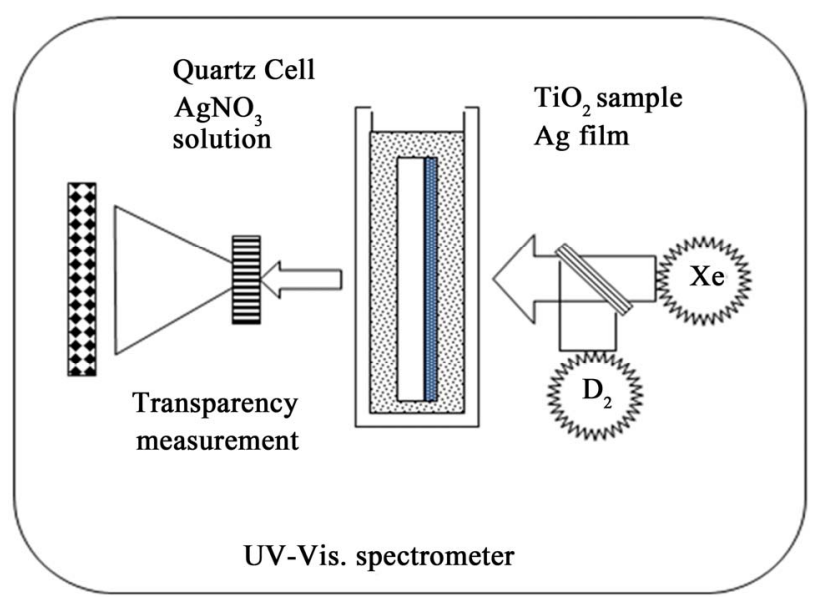

Figure 1. The schematic drawing of the photocatalytic activity measurement apparatus proposed in this study.

\section{Results and Discussion}

Figure 2 shows the $\mathrm{x}$-ray diffraction patterns of three kinds of $\mathrm{TiO}_{2}$ films coated on quartz glass substrates. An amorphous film (sample A: Figure 2(a)), a slightly crystallized film (sample B: Figure 2(b)) and a well-crystallized film (sample C: Figure 2(c)) were obtained, respectively. The $\mathrm{x}$-ray diffraction peaks of the sample $\mathrm{C}$ were assigned to those of the $\mathrm{TiO}_{2}$ with anatase and rutile structures. It was revealed that sample C was mainly crystallized into anatase structure and small amount of rutile structure was observable by x-ray diffraction. The sample B showed only one broad peak, which was assigned to the rutile structure. The photocatalytic activity measurement for coatings should be much more sensitive compared with that of the powder materials since the effective surface area of the coatings are much smaller than that of the powders. As a result, it has been very hard to evaluate the photocatalytic activities of the $\mathrm{TiO}_{2}$ coatings especially with poor crystalline quality (e.g. samples A and B) since the photocatalytic activities for those coatings are quite low.

Figure 3 shows the results of the photocatalytic activity measurement for the three samples (A, B and C) by the method described above. The transmittances of the visible light of the samples are plotted against UV irradiation time. The higher the photocatalytic activity of the sample is, the curve of the transmittance shifts to the lower left of the graph (shown by an arrow in Figure 3).

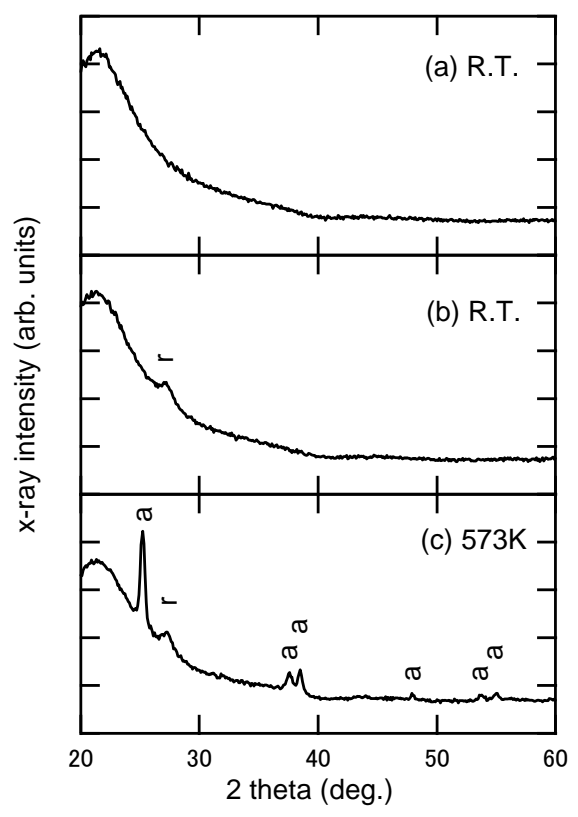

Figure 2. The $x$-ray diffraction patterns of three kinds of $\mathrm{TiO}_{2}$ films coated on quartz glass substrates. An amorphous film (sample A: (a)), a slightly crystallized film (sample B: (b)) and a well-crystallized film (sample C: (c)) were obtained, respectively. The letters " $a$ " and " $r$ " denote the anatase and rutile structures, respectively. 


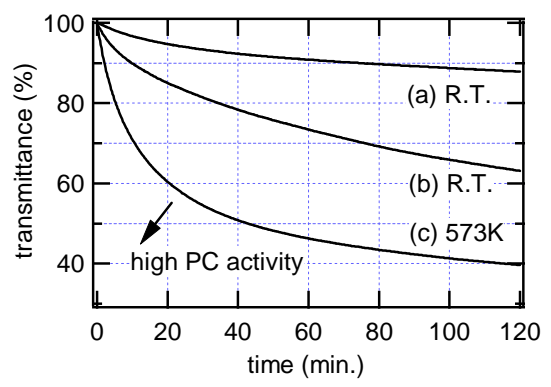

Figure 3. The results of the photocatalytic activity measurement for the three samples (A, B and C) obtained by the photocatalytic activity measurement apparatus proposed in this study. The transmittances of the visible light of the samples are plotted against UV irradiation time. The higher the photocatalytic activity of the sample is, the curve of the transmittance shifts to the lower left of the graph.

The transmittance curve of the sample $\mathrm{C}$ was observed at the lower left side of those of the samples A and B, reflecting the better crystalline quality of the sample C. Note that the photocatalytic activity of the amorphous sample (sample A) was measurable and a clear decrease in transmittance due to the Ag film formation on the sample surface was observed (Figure 3(a)) demonstrating the very high sensitivity for photocatalytic activity measurement of this method. It is also worth noticing that the photocatalytic activities of the amorphous sample (sample A) and the slightly crystallized sample (sample B) were clearly distinguishable (Figures 3(a) and (b)). Thus the high sensitivity combined with the wide dynamic range of this photocatalytic activity measurement was demonstrated.

\section{Conclusion}

A new photocatalytic activity measurement system based on the measurement of the photo-induced reduction of Ag ions was proposed. The feature of this system is to perform "ultraviolet irradiation for photocatalytic activation" and "Ag film thickness determination for photocatalytic activity evaluation" simultaneously and automatically. This method was applied on the $\mathrm{TiO}_{2}$ coatings on quartz glass substrates and was proven to be sensitive enough for evaluating the $\mathrm{TiO}_{2}$ coating with amorphous structure. Thus a photocatalytic activity measurement system with high sensitivity, wide dynamic range, easy operation and reproducibility, which is especially suited for the photocatalytic activity measurement of the coatings on transparent substrates, was realized.

\section{REFERENCES}

[1] A. Fujishima and K. Honda, "Electrochemical Photolysis of Water at a Semiconductor Electrode," Nature, Vol. 238, No. 5358, 1972, pp. 37-38. doi:10.1038/238037a0

[2] R. Wang, K. Hashimoto, A. Fujishima, M. Chikuni, E. Kojima, A. Kitamura, M. Shimohigoshi and T. Watanabe, "Light-Induced Amphiphilic Surfaces,” Nature, Vol. 388, No. 6641, 1997, pp. 431-432. doi:10.1038/40601

[3] N. Sakai, R. Wang, A. Fujishima, T. Watanabe and K. Hashimoto, "Effect of Ultrasonic Treatment on Highly Hydrophilic $\mathrm{TiO}_{2}$ Surfaces,” Langmuir, Vol. 14, No. 20, 1998, pp. 5918-5920. doi:10.1021/la980623e

[4] R. Wang, N. Sakai, A. Fujishima, T. Watanabe and K. Hashimoto, "Studies of Surface Wettability Conversion on $\mathrm{TiO}_{2}$ Single-Crystal Surfaces," The Journal of Physical Chemistry B, Vol. 103, No. 12, 1999, pp. 2188-2194. doi:10.1021/jp983386x

[5] M. Kamei and T. Mitsuhashi, "Hydrophobic Drawings on Hydrophilic Surfaces of Single Crystalline Titanium Dioxide: Surface Wettability Control by Mechanochemical Treatment," Surface Science, Vol. 463, No. 1, 2000, pp. L609-L612. doi:10.1016/S0039-6028(00)00635-X

[6] P. Frach, K. Goedicke, C. Gottfried and H. Bartzsch, “A Versatile Coating Tool for Reactive In-Line Sputtering in Different Pulse Modes,” Surface and Coatings Technology, Vol. 142-144, 2001, pp. 628-634. doi:10.1016/S0257-8972(01)01152-5

[7] S. Ohno, D. Sato, M. Kon, Y. Sato, M. Yoshikawa, P. Frach, Y. Shigesato, I. Tejedor-Tejedor and M. A. Anderson, "Photocatalytic Properties of $\mathrm{TiO}_{2}$ Films Deposited by Reactive Sputtering in Mid-Frequency Mode with Dual Cathodes," Japanese Journal of Applied Physics, Vol. 43, No. 12, 2004, pp. 8234-8241. doi:10.1143/JJAP.43.8234

[8] T. Miyagi, M. Kamei, T. Ogawa, T. Mitsuhashi, A. Yamazaki and T. Sato, "Pulse Mode Effects on Crystallization Temperature of Titanium Dioxide Films in Pulse Magnetron Sputtering," Thin Solid Films, Vol. 442, No. 1-2, 2003, pp. 32-35. doi:10.1016/S0040-6090(03)00934-9

[9] S. Nishimoto, B. Ohtani, H. Kajiwara and T. Kagiya, "Photoinduced Oxygen Formation and Silver-Metal Deposition in Aqueous-Solutions of Various Silver Salts by Suspended Titanium-Dioxide Powder," Journal of the Chemical Society, Faraday Transactions, Vol. 79, No. 11, 1983, pp. 2685-2694. doi:10.1039/f19837902685 